\title{
PRODUÇÃO, IMPORTAÇÃO, EXPORTAÇÃO E CONSUMO APARENTE DE PAINÉIS DE MADEIRA NO BRASIL ENTRE $1961 \mathrm{E}$ 2016
}

\author{
PRODUCTION, IMPORT, EXPORT AND APPARENT CONSUMPTION OF WOOD-BASED \\ PANELS IN BRAZIL DURING 1961-2016
}

\author{
Carlos Roberto Sanquetta ${ }^{1}$, Gabriel Mendes Santana ${ }^{2}$, Mateus Niroh Inoue Sanquetta ${ }^{3}$, \\ Thiago Wendling Gonçalves de Oliveira ${ }^{4}$, Ana Paula Dalla Corte ${ }^{5}$ \\ 1, 2, 3, 4, 5 Universidade Federal do Paraná, Curitiba, Paraná, Brasil - carlossanquetta@gmail.com, \\ gsantanaflorestal@gmail.com, mateus.sanquetta@gmail.com, thiagowendling@yahoo.com.br \& \\ anapaulacorte@gmail.com
}

\begin{abstract}
RESUMO
O segmento de painéis de madeira vem crescendo no Brasil com a consolidação das plantações florestais e da ampliação industrial. Este artigo aborda a evolução temporal da produção, importação, exportação e consumo aparente dos painéis de madeira no Brasil entre 1961 e 2016. Os dados foram extraídos do sistema FAOSTAT da FAO. Os produtos analisados foram agrupados em: chapas de fibras e duras, MDF+HDF, OSB, partículas, compensados, partículas e OSB e outros. Foram produzidas $218 \mathrm{M}$ t de painéis nesse interstício temporal, dos quais $38 \%$ de Compensados, 23\% de Partículas, 20\% de MDF+HDF, $10 \%$ de Chapas duras de fibras, $6 \%$ de Partículas+OSB, $2 \%$ de OSB e $1 \%$ de Outras. Historicamente Compensado foi o tipo de painel mais produzido no Brasil, mas atualmente MDF+HDF o superam (39\%), assim como Partículas (29\%). Todavia, o Compensado segue notoriamente como o principal produto de exportação na série histórica. As exportações superam as importações em quantidade, resultando em balanço positivo. O maior produto de importação é Chapas duras de fibras. O consumo aparente segue parcialmente as tendências da produção, mas como a maior parte do Compensado produzido no Brasil é para exportação, seu consumo interno atual é de apenas $6 \%$. O consumo per capita de painéis de madeira saltou de 3 em 1961 para $34 \mathrm{~m}^{3} \cdot$ hab $^{-1}$.ano-1 em 2016. Concluiu-se que os painéis de madeira desempenham importante papel na indústria florestal e que os produtos de partículas e fibras estão ganhando o mercado interno, enquanto os painéis laminados ainda são o carro-chefe nas exportações.
\end{abstract}

PALAVRAS-CHAVE: Chapas de madeira, Comércio, Madeira, Mercado, Produtos Florestais.

\begin{abstract}
The segment of wood-based panels has been growing in Brazil with the consolidation of forest plantations and industrial expansion. This paper discusses the temporal evolution of the production, imports, exports and apparent consumption of wood-based panels in Brazil between 1961 and 2016. The data were extracted from FAO's FAOSTAT system. The analyzed products were grouped as: fiber and hard density panels, MDF+HDF, OSB, particle, plywood, particles and OSB, and others. Total of $218 \mathrm{M}$ t of panels were produced in the period, of which $38 \%$ were of Plywood, 23\% Particles, 20\% MDF+HDF, 10\% Fiber and Hard Density, 6\% OSB+Particles, 2\% OSB and $1 \%$ of Others. Historically, Plywood was the most produced type of wood-based panel in Brazil, but currently MDF+HDF surpasses it (39\%), as well as Particles (29\%). However, Plywood is notoriously the main export product in the whole historical series. Exports surpass imports in quantity, resulting in a positive balance. The largest import product is Fiber and Hard Density. Apparent consumption partially follows production trends, but since most of the Plywood produced in Brazil is for export, its current domestic consumption is only $6 \%$. Per capita consumption of wood panels jumped from 3 in 1961 to $34 \mathrm{~m}^{3}$.hab he $^{-1}$.year ${ }^{-1}$ in 2016. It was concluded that wood-based panels play an important role in the forest industry and that particle and fiber products are gaining the domestic market, while laminated panels are still the flagship in exports.
\end{abstract}

KEYWORDS: Wood panels, Trade, Wood, Market, Forest Products. 


\section{INTRODUÇÃO}

Os países tropicais e subtropicais do Sudeste Asiático e da América do Sul, dentre eles o Brasil, tem as maiores produtividades de plantios florestais e os maiores investimentos nesse setor, apresentando taxas internas de retorno maiores do que $12 \%$, porém muitos desses países têm más políticas de regulação florestal e falta de conhecimento sobre os produtos gerados (CUBBAGE et al., 2010).

O Brasil possui a segunda maior área de florestas nativas no mundo e a nona posição entre os países com maior área de plantios florestais (FAO, 2015). O estoque volumétrico de madeira dessas florestas é de 95 e $2 \mathrm{G} \mathrm{m}^{3}$, respectivamente nas florestas nativas e plantadas (SANQUETTA et al., 2018a; SANQUETTA et al., 2018b).

Em decorrência disso o País vem se tornando um importante ator na economia florestal, figurando entre os maiores produtores e exportadores de produtos florestais em nível mundial. O Brasil encontra-se entre os maiores produtores de madeira para energia, serrados, celulose e papel e painéis (FAO, 2019).

O setor florestal brasileiro vem passando por transformações, migrando fortemente do extrativismo das florestas nativas para o seu manejo sustentável e, principalmente, para a expansão e fortalecimento da silvicultura. Diante disso, tem-se muito investimento tecnológico na produção de produtos florestais, dentre eles, os painéis de madeira (COLODETTE et al., 2014).

Os investimentos na silvicultura e na indústria baseada na madeira de plantios florestais atingiram cerca de $R \$ 6,5$ bilhões em 2018, dos quais $\mathrm{R} \$ 3,2$ bilhões em manejo florestal e $\mathrm{R} \$ 3,5$ bilhões na indústria. Com isso, a produção industrial chegou a 19,5 $\mathrm{M}$ t de celulose, 10,5 $\mathrm{M}$ t de papel, $7,9 \mathrm{M}$ t de painéis de madeira, $11 \mathrm{M}$ t de pisos laminados e 4,5 M t de carvão vegetal (IBÁ, 2018).

O segmento de painéis de madeira, em particular, é um dos mais destacados na produção florestal, sendo o Brasil o sétimo maior produtor mundial. Apesar dessa posição, outros países ainda estão muito à frente, notadamente a China, que produz cerca de $50 \%$ de todos painéis no mundo, enquanto o Brasil apenas 3\% (FAO, 2019).

Quanto ao consumo, o Brasil segundo BNDS (2014), ocupa a oitava posição, sendo o consumo de painéis maior relacionado com padrões culturais, hábitos e oferta local de produtos que com o próprio desenvolvimento do país, mesmo que a relação renda $x$ consumo seja maior para o caso dos painéis do que para os serrados.

A maior parte das indústrias de painéis de madeira reconstituída está nas regiões Sul e Sudeste do País, nos

Estados do Paraná, Santa Catarina, Rio Grande do Sul, São Paulo e Minas Gerais. Já as unidades fabris de painéis laminados estão localizadas nas regiões Sul e Amazônica, onde operam principalmente com toras do gênero Pinus spp. e de espécies tropicais (IBÁ, 2017).

Para orientar os investimentos no setor de base florestal é imprescindível que o País possua informações atualizadas e fidedignas sobre suas florestas e os segmentos industriais. O Sistema de Informações Florestais do governo federal, com gestão do Serviço Florestal Brasileiro (SFB), se constitui em um esforço de aperfeiçoamento das estatísticas florestais do País.

O SFB, como entidade representativa do Brasil nos organismos internacionais, aporta periodicamente dados ao sistema FAOSTAT, da FAO (Food and Agriculture Organization of the United Nations, Organização das Nações Unidas para Alimentação e Agricultura). Esse sistema congrega as mais diversas informações sobre produção agrícola, pecuária e de alimentos do mundo, entre as quais aquelas relacionadas aos produtos florestais (FAO, 2019).

A plataforma FAOSTAT disponibiliza os dados brutos de produção, exportação e importação de produtos florestais de muitos países, inclusive do Brasil. Contudo, tais informações ainda não foram muito exploradas cientificamente, existindo um número limitado de publicações que versam sobre a dinâmica temporal da produção e da comercialização de painéis de madeira no Brasil.

Desta forma, este trabalho objetivou analisar a produção, importação, exportação e consumo aparente de painéis de madeira no Brasil, agrupados em sete categorias, considerando a evolução temporal em um horizonte de 55 anos (1961 a 2016), desde que foi iniciada a série produzida pela FAO.

\section{MATERIAL E MÉTODOS}

Os dados utilizados neste estudo foram obtidos diretamente do site da FAO (http://www.fao.org/faostat/en/\#data/GF), no sistema FAOSTAT (FAO, 2019), contendo registros de produção, importação e exportação de vários tipos de painéis. Este trabalho avaliou os produtos conforme o grupamento (7 grupos) utilizado pela FAO, quais sejam:

- chapas duras de fibra

- MDF+HDF

- OSB

- partículas
- compensados

- partículas e OSB

- outros 
Para cálculo do consumo aparente anual de 1961 a 2016, foi utilizada a equação 1, que expressa a diferença entre a soma da produção e da importação menos a exportação:

$$
C A(i)=P(i)+I(i)-E(i)
$$

Em que: $C A(i)=$ consumo aparente dos painéis no ano $i[\mathrm{t}]$; $P(i)=$ produção no ano $i[\mathrm{t}] ; E(i)=$ exportação no ano $i[\mathrm{t}] ; \mathrm{e}$ $I(i)=$ importação no ano $i[\mathrm{t}]$.

Para o cálculo do consumo anual per capita (equação 2) foram utilizados dados populacionais do IBGE (IBGE, 2018):

$$
C A p c(i)=\left(\frac{C A(i)}{\operatorname{Pop}(i)}\right) / 1000
$$

Em que: $C A p c(i)=$ consumo aparente per capita dos painéis no ano $i\left[\mathrm{~kg} \cdot \mathrm{hab}^{-1}\right]$; e $\operatorname{Pop}(i)=$ população do Brasil no ano $i$ [node habitantes].

\section{RESULTADOS E DISCUSSÃO}

\section{Produção}

No período 1961 a 2016 (55 anos) foram produzidos $218 \mathrm{M} \mathrm{m}^{3}$ de painéis de madeira no Brasil, produzindo em termos percentuais no interstício temporal, 38\% de Compensados, 23\% de Partículas, 19\% de MDF+HDF, 10\% de Chapas duras de fibras, $6 \%$ de OSB+Partículas, $1 \%$ de OSB e $1 \%$ de Outras.

Atualmente (base 2016), a produção é de cerca de 10 $\mathrm{M} \mathrm{m}^{3}$.ano ${ }^{-1}$, sendo 39\% de MDF+HDF, 29\% de Partículas, 26 de Compensados, $3 \%$ de Chapas duras de fibras, $2 \%$ de OSB e menos de 1\% de Outras. Em 1961 a produção brasileira era de apenas 250 mil m$^{3}$ e mais de $75 \%$ da produção era de Compensados.

Historicamente o Compensado foi o tipo de painel mais produzido no Brasil, mas atualmente MDF+HDF e Partículas o superam, correspondendo a $39 \%$ e $29 \%$ da produção, respectivamente. Compensado representa, nos dias de hoje, $26 \%$ da produção nacional de painéis de madeira. $\mathrm{O}$ advento da fabricação de chapas de fibras e partículas de madeira mudou o perfil de produção e consumo no Brasil e esses painéis passaram a ganhar espaço alçando o topo após 2005.

A tendência geral de produção de painéis de madeira na atualidade é crescente, embora nos últimos anos, como reflexo da situação econômica desfavorável, a produção tenha estagnado (Figura 1).

\section{Importação}

A importação total de painéis de madeira no período de 55 anos foi de cerca de $26 \mathrm{M} \mathrm{t}$, com média anual de $468 \mathrm{mil}$ $\mathrm{m}^{3}$.ano ${ }^{-1}$. A maior parte desse volume é de chapas de fibras e duras, o que corresponde a $88 \%$ do montante importado na série histórica. Atualmente esse tipo de painel ainda é aquele com maior volume de importação (Figura 2).

Atualmente há uma tendência de queda nas importações de todos os tipos de painéis de madeira, com volume da ordem de $322 \mathrm{mil} \mathrm{m}^{3}$ em 2016. Essa queda é considerável, levando-se em conta que ao final de década de 1980 até meados da década de 1990 as importações tiveram média de mais de $600 \mathrm{mil} \mathrm{m}^{3}$.ano-1 ${ }^{-1}$ portanto, a queda nas importações representa praticamente $50 \%$.

\section{Exportação}

As exportações atingiram $71 \mathrm{M} \mathrm{m}^{3}$ no período de 1961 a 2016 , sendo $60 \%$ de Compensados e $32 \%$ de Chapas duras de fibras, ficando o restante com os demais tipos de produtos. Até meados da década de 1990 as exportações eram apenas de cerca de $500 \mathrm{mil} \mathrm{m}^{3}$.ano-1 . O grande salto das exportações de Compensados, e de painéis de madeira em geral no Brasil, por conseguinte, deu-se ao final de década de 1990 e início dos anos 2000, atingindo em 2005

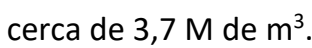

Após esse período houve uma queda abrupta nas exportações devido a fatores econômicos internacionais, chegando em 2011 a 1,7 M de $\mathrm{m}^{3}$. Todavia, a partir desse ano ocorreu uma retomada, atingindo em 2016 valores que se aproximam das quantias exportadas no auge de toda a série histórica, que atingiu 3,0 $\mathrm{M}$ de $\mathrm{m}^{3}$ (Figura 3).

\section{Consumo aparente}

No período de análise foram consumidos $172 \mathrm{M} \mathrm{m}^{3}$, o que representa $79 \%$ da produção. O produto de maior consumo foi o painel de Partículas, com $49 \mathrm{M} \mathrm{m}^{3}$, o que corresponde a $28 \%$ do consumo total de painéis no País (Figura 4). Em segundo lugar vieram Compensados e MDF+HDF, com 41 e $40 \mathrm{M} \mathrm{m}^{3}$, correspondendo a 24 e $23 \%$ do volume consumido em 55 anos, respectivamente. Chapas duras de fibras e OSB+Partículas representaram 13 e $8 \%$ do consumo, respectivamente, ficando o restante com os demais tipos de painéis.

No ano 2016 o consumo aparente maior foi de MDF+HDF, com 49\%, ou seja, 3,4 $\mathrm{M} \mathrm{m}^{3}$.ano-1. Em segundo lugar ficou o Compensado, com $38 \%$, isto é, $2,6 \mathrm{M} \mathrm{m}^{3}$.ano

${ }^{1}$. Desta forma, percebe-se uma mudança no padrão de 
consumo desses produtos, com migração das chapas laminadas (compensados) para MDF e HDF.

Evidencia-se tendência de crescimento do consumo doméstico de painéis, porém nos últimos anos (2013 em diante) houve certa retração, caindo de $8,8 \mathrm{M} \mathrm{m}^{3}$ para 7,7 $\mathrm{M} \mathrm{m}^{3}$.ano-1.

Houve um grande aumento na população do Brasil nesse período de 55 anos, passando de $73 \mathrm{M}$ de habitantes em 1961 a $204 \mathrm{M}$ em 2016. Considerando esse crescimento populacional e o consumo aparente de painéis de madeira, deduz-se que uma pessoa que consumia pouco mais de 3 $\mathrm{m}^{3}$ de painéis por ano em 1961, atualmente consome cerca de $34 \mathrm{~m}^{3}$. No início da década de 2010 esse valor chegou a mais de $44 \mathrm{~m}^{3}$.ano-1 ${ }^{-1}$ Computam-se nesse consumo aparente per capita $17 \mathrm{~m}^{3}$.ano-1 de chapas MDF+HDF e 13 $\mathrm{m}^{3}$.ano ${ }^{-1}$ de Partículas.

A produção de compensados advém de um número muito maior de indústrias espalhadas pelo País. Esse segmento se caracteriza pela existência de lacunas de informação em virtude da enorme pulverização do setor (BNDES, 2014). Segundo IBÁ (2017), a produção de compensados a partir de árvores plantadas atingiu 2,7 milhões de $\mathrm{m}^{3}$, dos quais 1,8 milhões foi destinado à exportação.

Desta forma, depreende-se que possa haver alguma inconsistência nos dados da produção desse produto, haja vista que esse é o montante reportado no FAOSTAT como a produção total de compensados do País. Em outras palavras, toda a produção de compensados de madeira tropical não está devidamente computada.

Segundo a ITTO (2017), o Brasil produziu $264 \mathrm{mil} \mathrm{m}^{3}$ de compensados de madeiras nativas tropicais em 2016, sendo $34 \mathrm{mil} \mathrm{m}^{3}$ destinados à exportação. Nenhuma importação desse produto foi verificada. Disso deduz-se que que a produção de compensados advinda de madeira tropical representa menos de $10 \%$ do total desse produto fabricado no País. Portanto, mais de $90 \%$ do volume de compensados atualmente são produzidos a partir de madeira de plantações florestais, sobretudo de coníferas do gênero Pinus spp. e, em muito menor escala, de Schizolobium amazonicum Huber ex Ducke (paricá).

Adicionalmente, o percentual exportado de compensados de madeiras tropicais, em volume, é de apenas $1,5 \%$. Assim, sendo, com base nesses números, pode-se afirmar que a maior parte do compensado de madeiras nativas tropicais é consumido internamente no País.

Os resultados deste estudo mostram que tem havido uma expansão da produção, exportação e consumo de painéis de madeira no Brasil em 55 anos. Particularmente esse segmento ganhou força a partir de meados da década de 1990 com a ampliação das exportações de compensados, que tiveram grande salto. Essa expansão deu-se em favor dos painéis produzidos a partir de madeira de plantações florestais, que também ampliaram muito suas áreas de plantio no período (SANQUETTA et al., 2018a).

Segundo Colodette et al. (2014), o maior crescimento em produtos de madeira no Brasil é dos painéis industrializados (MDF, MDF e OSB) de plantios de eucalipto, principalmente graças à boa performance do setor de construção na última década. Contudo investimentos e políticas ainda são necessários para manter a competitividade econômica e desenvolvimento social no País.

Houve também mudança de padrão de consumo pelos brasileiros, que passaram a consumir mais móveis, componentes domésticos e de construção à base de chapas de madeira reconstituída, que vem crescendo a taxas elevadas no País, sobretudo o MDF, mas também o MDP. A média mundial de crescimento nos últimos anos é $4,7 \%$ ao ano (BNDES, 2014). Relações socioeconômicas da população são importantes preditores do comportamento e consumo dos produtos de madeira, e devem ser verificadas para guiar projetos de manejo e conservação das florestas (MEDEIROS et al., 2012).

Se de um lado a competitividade dos painéis de madeira reconstituída e compensados produzidos a partir de madeira de florestas plantadas vem se consolidando, o mesmo não se verifica em relação aos produtos de madeira nativa tropical. Estudos apontam que a madeira compensada (madeira serrada) de florestas tropicais vem perdendo competitividade nas últimas duas décadas.

Esta perda de competitividade está diretamente relacionada à crescente dificuldade de explorar e vender madeira tropical da Amazônia, bem como aos custos de produção no Brasil, principalmente devido ao custo de energia, logística e dificuldades de crédito no país (SOUZA et al., 2018).

Portanto, o que se espera para o futuro próximo é o crescimento maior da indústria de painéis de madeira fabricados a partir de fibras e partículas e uma ocupação de nichos específicos de mercado que demandam chapas compensadas. Isso tem implicações importantes no manejo das florestas, pois este deverá ser impactado no tocante à escolha da densidade de plantio, realização ou não de podas e desbastes, melhoramento genético, bem como a redução na idade de rotação ou ciclo de corte. 


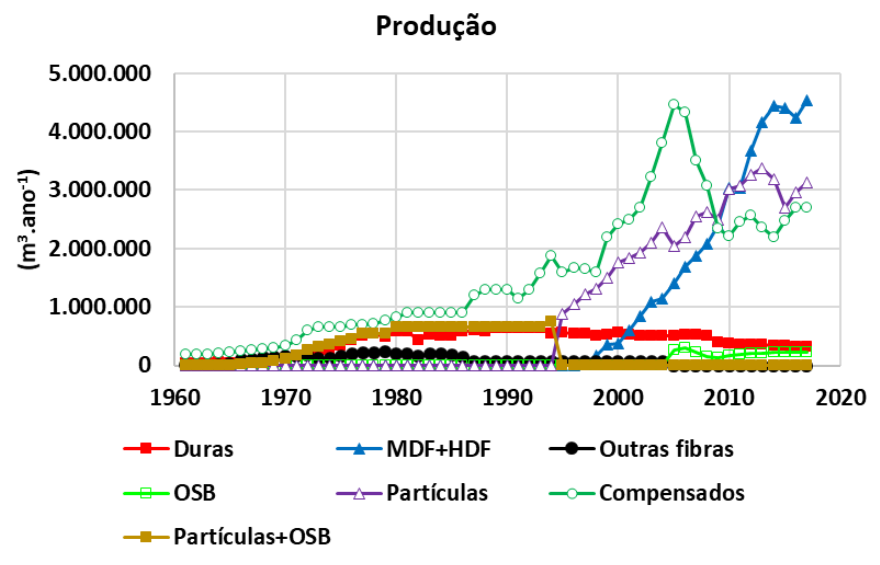

Figura 1. Produção de painéis de madeira no Brasil entre 1961 e 2016 (adaptado de FAO, 2019).

Exportação

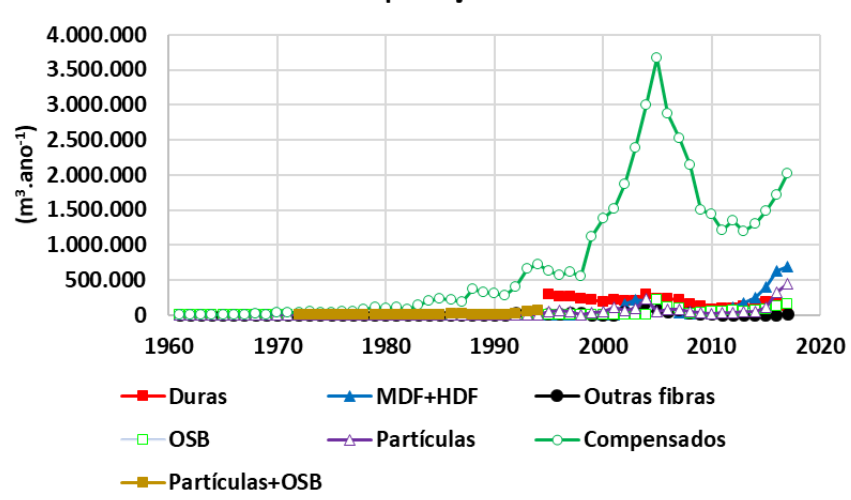

Figura 3. Exportação de painéis de madeira no Brasil entre 1961 e 2016 (adaptado de FAO, 2019).

\section{CONCLUSÕES}

- Houve um grande crescimento da produção de painéis de madeira no período de análise no Brasil, particularmente de MDF+HDF e Partículas.

- Historicamente Compensado é o tipo de painel mais produzido no Brasil, mas atualmente MDF+HDF e Partículas já o superam.

- As exportações superam as importações em quantidade, resultando em balanço positivo.

- Compensado continua sendo o principal produto de exportação na série histórica e o maior produto de importação é Chapas Duras de Fibras.

- Os painéis de maior consumo doméstico são MDF+HDF e Partículas, os quais substituíram em grande parte os usos de Compensados no Brasil.

- Houve um grande aumento no consumo per capita de painéis de madeira em 55 anos, de quase $1.000 \%$.

- Produtos de partículas e fibras estão ganhando o mercado interno, enquanto painéis compensados ainda são o carro-chefe nas exportações.

- Os painéis de madeira desempenham importante papel na indústria de base florestal.

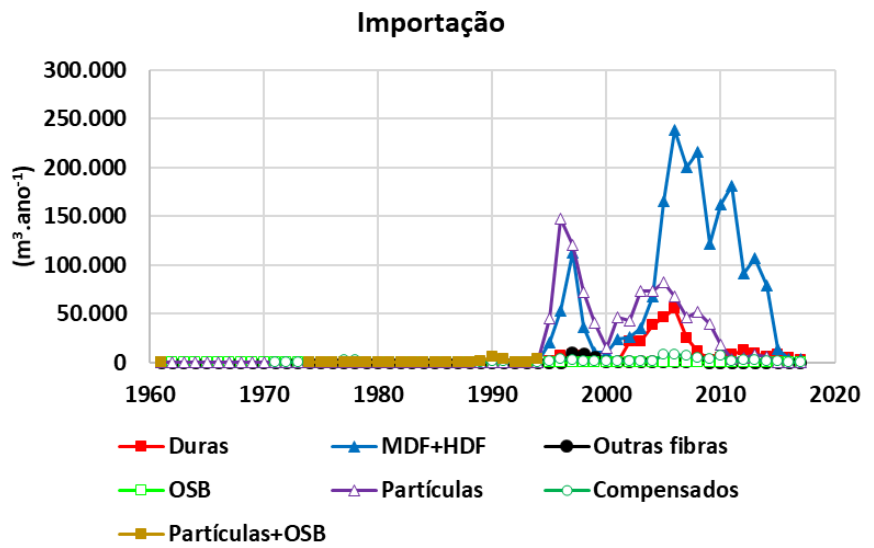

Figura 2. Importação de painéis de madeira no Brasil entre 1961 e 2016 (adaptado de FAO, 2019).

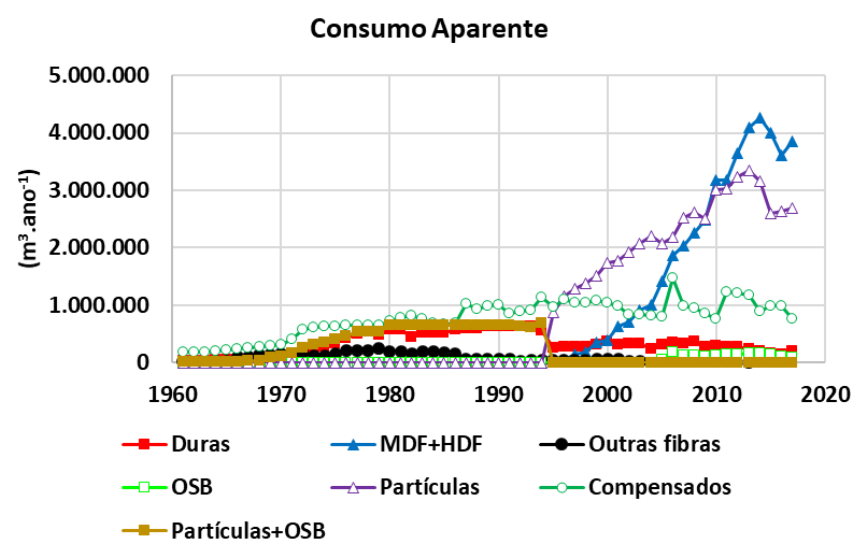

Figura 4. Consumo aparente de painéis de madeira no Brasil entre 1961 e 2016 (adaptado de FAO, 2019).

\section{AGRADECIMENTOS}

À CAPES pelo suporte financeiro a este trabalho, por meio de bolsa de Pesquisador Estágio Sênior de PósDoutorado no Exterior ao primeiro autor junto à Universidade de Lisboa, Portugal. Apoio: Coordenação de Aperfeiçoamento de Pessoal de Nível Superior - Brasil (CAPES) - Código de Financiamento 001

\section{REFERÊNCIAS}

BNDES - BANCO NACIONAL DE DESENVOLVIMENTO ECONÔMICO E SOCIAL. Panorama de mercado: painéis de madeira. 2014. Disponível: http://www.bndes.gov.br/bibliotecadigital

COLODETTE, J.L. et al. The Brazilian wood biomass supply and utilization focusing on eucalypt. Chemical and Biological Technologies in Agriculture, v.1, n.25, p.1-8, 2014.

CUBBAGE, F. et al. Global timber investments, wood costs, regulation, and risk. Biomass and bioenergy, v.34, p.1667-1678, 2010.

FAO - FOOD AND AGRICULTURE ORGANIZATION OF THE UNITED NATIONS. Global Forest Resources Assessment 2015. Rome: 
2015. Disponível em: http://www.fao.org/3/a-i4808e.pdf

FAO - FOOD AND AGRICULTURE ORGANIZATION OF THE UNITED

NATIONS. FAOSTAT. 2019. Disponível em:

http://www.fao.org/faostat/en/\#data/GF

IBÁ - INSTITUTO BRASILEIRO DE ÁRVORES. Relatório 2017. São Paulo: IBÁ, 2018.

IBÁ - INSTITUTO BRASILEIRO DE ÁRVORES. Relatório 2016. São Paulo: IBÁ, 2017.

ITTO - INTERNATIONAL TROPICAL TIMBER ORGANIZATION. Biennial Review and Assessment of the World Timber Situation

2015-2016. 2017. Disponível em:

https://www.itto.int/news_releases/id=5195

MEDEIROS, P.M. et al. Socio-economic predictors of domestic wood use in an Atlantic forest area (north-east Brazil): a tool for directing conservation efforts. International Journal of Sustainable Development \& World Ecology, v.19, n.2, p.189-195, 2012.

SANQUETTA, C.R. et al. Dinâmica em superfície, volume, biomassa e carbono nas florestas plantadas brasileiras: 19902016. BIOFIX Scientific Journal, v.3, n.1, p.152-160, 2018 a.

SANQUETTA, C.R. et al. Dinâmica em superfície, volume, biomassa e carbono nas florestas nativas brasileiras: 1990-2015. BIOFIX Scientific Journal, v.3, n.1, p.193-198, 2018b.

SOUZA, S.N. et al. Competitiveness of Brazilian tropical wood on the international market. Floresta e Ambiente, v.25, n.1, p.e20150218, 2018.

Recebido em 18-04-2019 Aceito em 30-07-2019 